\title{
Effective absorption coefficient and effective thickness in attenuated total reflection spectroscopy
}

\author{
A. Mendoza-Galvan, J. G. Mendez-Lara, R. A. Mauricio-Sanchez, Kenneth Järrendahl \\ and Hans Arwin
}

The self-archived postprint version of this journal article is available at Linköping University Institutional Repository (DiVA):

http://urn.kb.se/resolve?urn=urn:nbn:se:liu:diva-174385

N.B.: When citing this work, cite the original publication.

Mendoza-Galvan, A., Mendez-Lara, J. G., Mauricio-Sanchez, R. A., Järrendahl, K., Arwin, H., (2021), Effective absorption coefficient and effective thickness in attenuated total reflection spectroscopy, Optics Letters, 46(4), 872-875. https://doi.org/10.1364/OL.418277

Original publication available at:

https://doi.org/10.1364/OL.418277

Copyright: Optical Society of America

http://www.osa.org/ 


\title{
Effective absorption coefficient and effective thickness in attenuated total reflection spectroscopy
}

\author{
A. Mendoza-Galván, ${ }^{1,}{ }^{*}$ J. G. Méndez-Lara, ${ }^{1}$ R. A. Mauricio-SÁnchez, ${ }^{1}$ \\ K. JÄRRENDAHL, ${ }^{2}$ H. ARWIN ${ }^{2}$ \\ ${ }^{1}$ Cinvestav-Unidad Querétaro, Libramiento Norponiente 2000, Querétaro, 76230 Mexico \\ ${ }^{2}$ Materials Optics, Department of Physics, Chemistry and Biology, Linköping University, SE-58183 Linköping, Sweden \\ *Corresponding author: amendoza@cinvestav.mx
}

Received XX Month XXXX; revised XX Month, XXXX; accepted XX Month XXXX; posted XX Month XXXX (Doc. ID XXXXX); published XX Month XXXX

\begin{abstract}
Since the introduction of attenuated total reflection (ATR) spectroscopy for the characterization of materials, attempts have been made to relate the measured reflectivity $(R)$ to the absorption coefficient $(\alpha)$ of the absorbing material of interest. The common approach is limited to the low absorption case under the assumption $R \sim \exp \left(-\alpha d_{\mathrm{e}}\right)$ where $d_{\mathrm{e}}$ is an effective thickness, which is evaluated for the lossless case. In this letter, a more detailed derivation leads to $R=\exp \left(-\beta d_{\mathrm{p}} / 2\right)$ enabling the definition of an ATR effective absorption coefficient $\beta$ and the penetration depth $d_{\mathrm{p}}$ of the electric field in the absorbing material. It is found that $\beta \sim \pi \varepsilon_{2} / \lambda$, where $\varepsilon_{2}$ is the imaginary part of the complex dielectric function of the absorbing material and $\lambda$ is the wavelength. An alternative formulation is $R=\exp \left(-\alpha d_{\mathrm{ef}}\right)$ where $d_{\mathrm{ef}}$ is a generalized effective thickness for arbitrary strength of absorption which reduces to $d_{\mathrm{e}}$ in the low absorption limit. Experimental data for water, the biopolymer chitosan, and soda-lime glass prove the reliability of the ATR-effective absorption coefficient in the infrared range. (c) 2020 Optical Society of America
\end{abstract}

Total internal reflection is a phenomenon for radiation incident from an optically denser medium with refractive index $n_{a}$, impinging the boundary ( $x y$ plane) with an optically rarer medium with refractive index $n<n_{\mathrm{a}}$, at an angle of incidence $\theta$ larger than the critical angle given by $\theta_{c}=\sin ^{-1}\left(n / n_{a}\right)$. Under these conditions the reflectivity for parallel $\left(R_{p}\right)$ and perpendicular $\left(R_{s}\right)$ polarization both equals unity. However, to satisfy the boundary conditions an evanescent wave exists in the rarer medium with an electric field decaying as $\exp \left(-z / d_{\mathrm{p} 0}\right)$ perpendicular $(z$ direction) to the boundary. The penetration depth $d_{\mathrm{p} 0}$ is wavelength $(\lambda)$ dependent and given by [1],

$$
d_{\mathrm{p} 0}=\frac{\lambda}{2 \pi \sqrt{n_{\mathrm{a}}^{2} \sin ^{2} \theta-n^{2}}}
$$

Sixty years ago, Fahrenfort introduced attenuated total reflection (ATR) to investigate the infrared response of organic compounds [1]. He noticed that for absorbing materials characterized by a complex refractive index $N=n+i k$, where $k$ is the extinction coefficient, the internal reflection spectrum $R$ resembles that obtained in transmission mode in spectral regions where $k \neq 0$. To highlight the application of ATR measurements for quantitative purposes, Fahrenfort introduced the "formal optical density" as $\log R^{-1}$.

Since ATR measurements are very sensitive and particularly advantageousfor thick samples for which transmittance measurements are not possible, $\log R^{-1}$ was commonly used as a representation for an absorption spectrum. For low absorption $(k<0.1)$ Hansen developed a series expansion of $R_{\mathrm{p}}$ and $R_{\mathrm{s}}$ to investigate the range of applicability of the Beer-Lambert law in ATR measurements [2]. The concept of "effective thickness" $d_{\text {e }}$ was introduced for low absorption as the thickness of a film of the same material which would give the same absorption for transmission at normal incidence [3]. That is, if reflection losses are neglected, the transmittance of a film of thickness $d$ and absorption coefficient $\alpha=4 \pi k / \lambda$ has a dependence $T \sim \exp (-\alpha d) \approx 1-\alpha d$. Analogously, for ATR measurements it was assumed a similar form $R \sim \exp \left(-\alpha d_{\mathrm{e}}\right) \approx 1-\alpha d_{\mathrm{e}}$ where $d_{\mathrm{e}}$ is given by $d_{\mathrm{e}}=\left(d_{\mathrm{e}, \mathrm{s}}+d_{\mathrm{e} p}\right) / 2$. Here, $d_{\mathrm{e}(\mathrm{p}, \mathrm{s})}$ are effective thicknesses calculated [3] for s-polarization,

$$
d_{\mathrm{e}, \mathrm{s}}=\frac{2 n_{\mathrm{a}} n \cos \theta}{\left(n_{\mathrm{a}}^{2}-n^{2}\right)} d_{\mathrm{p} 0}
$$

and p-polarization,

$$
d_{\mathrm{e}, \mathrm{p}}=\frac{2 n_{\mathrm{a}} n \cos \theta\left(2 n_{\mathrm{a}}^{2} \sin ^{2} \theta-n^{2}\right)}{\left(n_{\mathrm{a}}^{2}-n^{2}\right)\left(n_{\mathrm{a}}^{2} \sin ^{2} \theta-n^{2} \cos ^{2} \theta\right)} d_{\mathrm{p} 0},
$$

Since it is assumed that $R \sim \exp \left(-\alpha d_{\mathrm{e}}\right.$ ), the ATR-formal optical density (or absorbance) $\log R^{-1}$ is usually corrected by dividing with the effective thickness $d_{\mathrm{e}}$. Here "correction" means to transform the experimental $\log R^{-1}$ spectrum into a quantity mimicking the absorption coefficient. 
Despite the limitation of the zero-absorption assumption, $k=0$ in Eqs. (2) and (3), the effective thickness approach has been successfully applied over the years to investigate the ATR infrared response and structure of proteins, lipids, biological membranes [4-6], depth profiling (taking advantage of the dependence of $d_{\mathrm{p} 0}$ on $n_{\mathrm{a}}$ and/or $\theta$ in Eq. (1)) [7], soft matter at interfaces [8], imaging of biological systems and biopharmaceuticals $[9,10]$. In most cases, $n$ is assumed to be constant and only in a few applications dispersion is consider [5,11].

However, a long time ago Müller et al. remarked that even for $\theta>\theta_{c}$ electromagnetic waves propagate in the absorbing medium but with planes of constant amplitude parallel to the interface non coincident with planes of constant phase [12], Thus, in terms of the dielectric constant of the denser material $\varepsilon_{\mathrm{a}}=n_{\mathrm{a}}{ }^{2}$, and the real $\varepsilon_{1}$ and imaginary $\varepsilon_{2}$ parts of the complex dielectric function $\varepsilon=\varepsilon_{1}+i \varepsilon_{2}\left(=N^{2}\right)$ of the sample, the penetration depth is given by [12],

$$
d_{\mathrm{p}}=\frac{\lambda}{\sqrt{2} \pi\left[\sqrt{\left(\varepsilon_{\mathrm{a}} \sin ^{2} \theta-\varepsilon_{1}\right)+\sqrt{\left(\varepsilon_{\mathrm{a}} \sin ^{2} \theta-\varepsilon_{1}\right)^{2}+\varepsilon_{2}^{2}}}\right]} \text { (4) }
$$

Notice that Eq. (4) reduces to Eq. (1) for a non-absorbing material, i.e., when $\varepsilon_{2}=0$ and thus also $k=0$. The relevance of Eq. (4) to describe absorption in ATR measurements will be demonstrated.

Other approaches to analyze ATR spectra use either Kramers-Kronig transforms [13-15] or Lorentz dispersion models to determine $\varepsilon$ or $N$ [16]. However, these procedures require a nearly perfect contact at the interface (complicated to achieve) for accurate determination of optical functions including absorption.

The objective of this letter is to provide a general relationship to describe ATR spectra in terms of fundamental quantities accounting for absorption, i.e., $\varepsilon 2, k$, and/or $\alpha$. The formalism is applied to experimental data for chitosan, which is a derivative of the biopolymer chitin. The latter is found in the shell of crustaceous, fungi, and the cuticle of beetles [17]. The interest in chitosan is because it has found applications in diverse fields and often it is characterized with ATR in the infrared range $[18,19]$. Data for water and soda lime glass are also included as contrasting examples of lower refractive index and stronger absorption, respectively.

Assuming unpolarized incident radiation at the interface between two media, one non-absorbing $\left(n_{\mathrm{a}}\right)$ and the other absorbing $(N)$, algebraic manipulation of $R=\left(R_{p}+R_{s}\right) / 2$ as shown in Supplement 1 leads to,

$$
R=\frac{1-u}{(1+u)^{2}}
$$

where,

$$
u=\frac{2 n_{\mathrm{a}} z_{1} \cos \theta \sqrt{\varepsilon_{\mathrm{a}} \sin ^{4} \theta+c \cos ^{2} \theta}}{\sqrt{\varepsilon_{\mathrm{a}} \cos ^{2} \theta+c} \sqrt{\cos ^{2} \theta\left(\varepsilon_{\mathrm{a}} \sin ^{2} \theta-c\right)^{2}+c \varepsilon_{\mathrm{a}}}} .
$$

The expressions for $z_{1}$ and $c$ are given in Eqs. (S9) and (S10) in Supplement 1, respectively. As shown in Supplement 1, the natural logarithm of Eq. (5) in combination with relations between $\ln$ and tanh ${ }^{-1}$ functions lead to an exponential dependence of $R$ on $\alpha$ for arbitrary absorption given by,

$$
R=\exp \left(-\alpha d_{\mathrm{ef}}\right)
$$

where the effective thickness $d_{\mathrm{ef}}$ is given by,

$$
d_{\mathrm{ef}}=\left(\tanh ^{-1} u+\tanh ^{-1} \frac{u}{2+u}\right) \frac{n d_{\mathrm{p}}}{z_{1}} .
$$

Both $z_{1}$ and $d_{\mathrm{p}}$ depend on $\varepsilon_{2}$, and thus also $d_{\mathrm{ef}}$ in Eq. (8) which, although undesired, is unavoidable. Furthermore, Eq. (7) can alternatively be viewed in terms of an effective ATR absorption coefficient $\beta$ defined by,

$$
R=\exp \left(-\beta d_{\mathrm{p}} / 2\right)
$$

where $d_{\mathrm{p}} / 2$ is used because $R$ involves measurements of irradiance and the intensity decays as $I \sim \exp \left(-2 z / d_{\mathrm{p}}\right)$.

Since the dependence of $d_{\mathrm{ef}}$ (and $\beta$ ) on fundamental quantities in Eq. (8) are complicated, it is convenient to look for a series expansion of tanh $^{-1}$ as shown in Eqs. (S15) and (S16) in Supplement 1, leading to,

$$
\ln R^{-1}=2 u\left[1+\frac{1}{2+u}+\frac{1}{3} u^{2}+\frac{1}{3} \frac{u^{2}}{(2+u)^{3}}+O\right]
$$

where $O$ stands for terms of higher order (see Eq. (S22) in Supplement 1). The rapid convergence of the series in Eq. (10) makes possible to approximate the calculations with good accuracy by using a first few terms. Indeed, for $u=0.5$ the difference between $R$ calculated as in Eq. (5) and up to the third term in Eq. (10) is only 0.005 (Fig. S3 in Supplement 1 ), which is within the experimental error. Furthermore, the values of $u$ can be controlled by choosing adequately the experimental settings $\left(n_{\mathrm{a}}\right.$ and $\theta$ ) as is shown below. From Eqs. (9) and (10) and $d_{\mathrm{p}}=\lambda /\left(2 \pi z_{2}\right)$ we get,

$$
\beta=\frac{2 \ln R^{-1}}{d_{\mathrm{p}}}=\frac{4 \pi}{\lambda} \varepsilon_{2} 2 f s,
$$

where $f=u /\left(2 z_{1}\right)$ is determined from Eq. (6), $s$ is the summation of the first three terms in brackets in Eq. (10), and $\varepsilon_{2}=2 z_{1} z_{2}$ ( $z_{2}$ is given in Eq. (S9) in Supplement 1). Thus, an explicit exponential dependence of $R$ on $\varepsilon 2$ has been accomplished which highlights the importance of using Eq. (4). Furthermore, substitution of $\varepsilon_{2}=2 n k$ in Eq. (11) leads to the exponential form $R=\exp \left(-\alpha d_{\mathrm{ef}}\right)$ as in Eq. (7) where the effective thickness is now expressed as,

$$
d_{\mathrm{ef}}=2 n f s d_{\mathrm{p}} .
$$

Since the derivations above have no restrictions on the strength of absorption, Eq. (12) is a generalization of the effective thickness for low absorption. The relationship between $d_{\mathrm{e}}$ and $d_{\mathrm{ef}}$ in the limit $k \rightarrow 0\left(\left.d_{\mathrm{ef}}\right|_{k=0}\right)$ is obtained in Eqs. (S24)-(S31) in Supplement 1 leading to,

$$
\frac{d_{\mathrm{e}}}{\left.d_{\mathrm{ef}}\right|_{k=0}}=1+\frac{1}{3} \frac{\left(n / n_{\mathrm{a}}\right)^{2} \cos 2 \theta}{\sin ^{2} \theta-\left(n / n_{\mathrm{a}}\right)^{2} \cos ^{2} \theta} .
$$

Clearly $d_{\mathrm{e}}=\left.d_{\mathrm{ef}}\right|_{k=0}$ at $\theta=45^{\circ}$ regardless of $n_{\mathrm{a}}$ and $n$. At larger angles of incidence up to $60^{\circ}$, the deviation is smaller than 0.1 for an interface with diamond for common values of the ratio $n / n_{\text {a. }}$. Using a crystal with higher refractive index, e.g., of germanium (Ge), the deviation is even smaller (Fig. S4 in Supplement 1). To illustrate the role of the effective parameters in ATR spectroscopy, experimental data are analyzed for representative liquid, polymeric, and inorganic media as shown below.

Figure 1(a) shows the experimental and calculated (Eq. 10) $\ln R^{-1}$ spectra of deionized water. It is expected that the contact of a liquid with diamond offers an ideal interface. As specified by the supplier (PIKE) of the ATR accessory, the refractive index of the diamond crystal is $n_{\mathrm{a}}=2.4$ and nominal angle of incidence $45^{\circ}$. However, the actual value of $\theta$ should be determined because internal mirrors in the accessory must 
be routinely adjusted to get the maximum intensity in the background spectrum. In the present case, a good match of the experimental and calculated spectra in Fig. 1(a) was achieved for $\theta=49^{\circ}$, using reported optical constants of water [20]. This value of $\theta$ serve as the calibrated value for further measurements. Figure 1(b) shows the effective ATR absorption coefficient calculated from Eq. (9) using the experimental reflectivity spectrum $R_{\exp }$, i.e., $\beta_{\exp }=2 \ln R \exp ^{-1} / d_{\text {p }}$. Furthermore, for the experimental settings, the value of $u(<0.3)$ is small enough to approximate the series in Eq. (10) up to the third term (Fig. S5 in Supplement 1). Since the sum of $2^{\text {nd }}$ and $3^{\text {rd }}$ terms as well as $f$ are smoothly dependent on wavenumber, they can be approximated by their mean values 0.49 and 0.383 , respectively. Thus, from Eq. (11) $\beta \approx 1.14 \beta 0$ where $\beta_{0}=4 \pi \varepsilon_{2} / \lambda$. Figure $1(\mathrm{~b})$ shows that it is indeed a very good approximation. In Fig. 1(b) the difference between $\beta$ and $1.14 \beta$ at the maxima which are located at 700,1640, and $3360 \mathrm{~cm}-1$ is $6.4,2.3$, and $7.6 \%$, respectively.

The characteristic lengths in Eqs. (1), (4), (8) and the average of Eqs. (2) and (3) for the diamond-water interface, decrease from about 2 to $0.36 \mu \mathrm{m}$ in the spectral range considered as seen in Fig. 1(c). The spectrum of $k$ for water shows three maxima of $0.41,0.131$, and 0.285 at 650,1640 , and $3400 \mathrm{~cm}^{-1}$, respectively. As can be noticed, in most of the spectral range investigated the penetration depths are $d_{\mathrm{p}} \approx d_{\mathrm{p} 0}$ and $d_{\mathrm{e}} \approx d_{\mathrm{ef}}$ for the effective thicknesses. However, $d_{\mathrm{e}}>d_{\mathrm{ef}}$ in the range 650 $900 \mathrm{~cm}^{-1}$ as is shown in the insert of Fig. 1(c), indicating the limitation of using Eqs. (2) and (3) in this range. For example, the difference at 650 $\mathrm{cm}^{-1}$ is $16.3 \%$. In the insert, that difference is apparently larger because the range of the vertical axis is limited from 1.2 to $2.8 \mathrm{um}$.

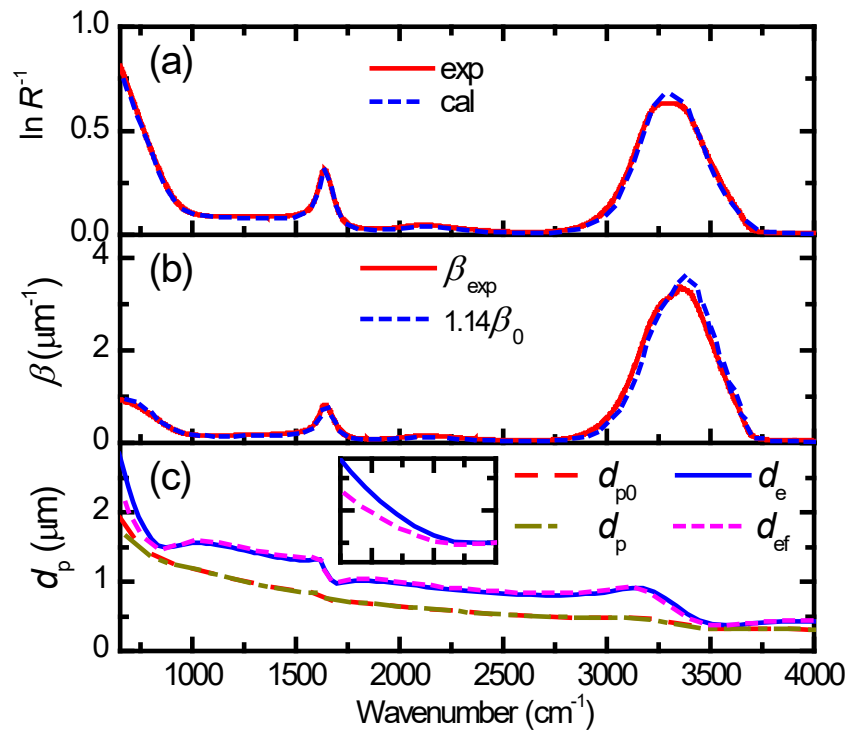

Fig. 1. (a) $\ln R^{-1}$ spectra of experimental $R$ and data calculated with Eq. (5) at $\theta=49^{\circ}$ for a diamond-water interface using reported optical constants of water [20]. (b) Effective ATR absorption coefficient $\beta_{\mathrm{exp}}=2 \ln R_{\exp ^{-1}} / d_{\mathrm{p}}$ compared to $1.14 \beta_{0}=(1.14) 4 \pi \varepsilon_{2} / \lambda$. (c) Penetration depths from Eqs. (1) and (4), effective thicknesses from Eq. (8) and $d_{\mathrm{e}}$ $=\left(d_{\mathrm{e}, \mathrm{s}}+d_{\mathrm{e}, \mathrm{p}}\right) / 2$, the average of Eqs. (2) and (3). The insert in (c) shows $d_{\mathrm{e}}$ and $d_{\mathrm{ef}}$ in the range $650-900 \mathrm{~cm}^{-1}$.

The second example is ATR measurements on a film of the biopolymer chitosan with thickness $4.32 \pm 0.03 \mu \mathrm{m}$. Its complex dielectric function was determined by combining oblique spectroscopic ellipsometry (IRSE, J. A. Woollam Co., Inc.) and normal incidence transmittance irradiance measurements (Spectrum GX, Perkin Elmer), as presented in Figs. S1 and S2, respectively, in Supplement 1. The chitosan films were cast following the procedure reported in our previous works [21,22]. Further experimental details are given in Supplement 1.

Figure 2(a) shows experimental and calculated $\ln R^{-1}$ spectra for a chitosan film. The calculated spectrum has been multiplied by 0.74 to put data in the same scale as the experimental spectrum. As is known, in ATR measurements the crystal and solid samples are brought in physical contact. Forcing the contact often improves the quality of ATR spectra. To avoid damage on the crystal, the pressure clamp limits the maximum pressure applied. This affects the optical contact between the crystal and the sample and therefore the magnitude of $R$. The presence of interfacial roughness creating air gaps decreases the penetration depth to an effective value between that of $d_{\mathrm{p}}$ in Eq. (4) and that of vacuum, $d_{\mathrm{v}}$ in Eq. (1) with $n_{\mathrm{a}}=1$. Other methods to increase the magnitude of an ATR spectrum include the use of index matching liquids or solids [23]. Furthermore, air gaps produce smaller values of $\varepsilon_{2}$ suggesting the use of an effective complex dielectric function. In a first approximation, the overall result is that experimental and calculated $\ln R^{-1}$ data, agree within a scale factor $(0.74$ in the present case of the diamond-chitosan interface). A rigorous treatment of this subject is beyond the scope of this letter.

Figure 2(b) shows the effective ATR absorption coefficient $\beta_{\exp }$ calculated from Eq. (9) using the experimental reflectivity spectrum. Maxima in $\varepsilon_{2}$ locate resonant frequencies among which the most important are vibrations of the glycosidic ring in range $800-1100 \mathrm{~cm}^{-1}$, the glycosidic bond at $1150 \mathrm{~cm}^{-1}$, amino and amide bands at 1500-1700 $\mathrm{cm}^{-1}, \mathrm{CH}$ about $2900 \mathrm{~cm}^{-1}$, and O-H stretching modes in the range 3000 $3500 \mathrm{~cm}^{-1}$ [21]. Substitution of the mean values $s=1.486$ and $f=0.454$ (Fig. S6 in Supplement 1), in Eq. (11) gives $\beta \approx 1.35 \beta 0$. As can be seen in Fig. 2(b). At $1077 \mathrm{~cm}^{-1}$ the difference between $\beta$ and $\beta_{0}$ is $22.6 \%$.

As expected from Eq. (13), Fig. 2(c) shows that the penetration depths $d_{\mathrm{p}} \approx d_{\mathrm{p} 0}$ and the effective thickness $d_{\mathrm{e}} \approx d_{\mathrm{ef}}$ in spectral ranges of low absorption. That corresponds to wavenumbers outside the range $900-1100 \mathrm{~cm}^{-1}$ of vibrations belonging to the glycosidic ring. The stronger absorption around $1000 \mathrm{~cm}^{-1}$ in chitosan produces smaller values on $d_{\mathrm{p}}$ and $d_{\mathrm{ef}}$ than on $d_{\mathrm{p} 0}$ and $d_{\mathrm{e}}$ as calculated with Eqs. (1) and (2) and should be considered for interpretation of data for depth profiling.

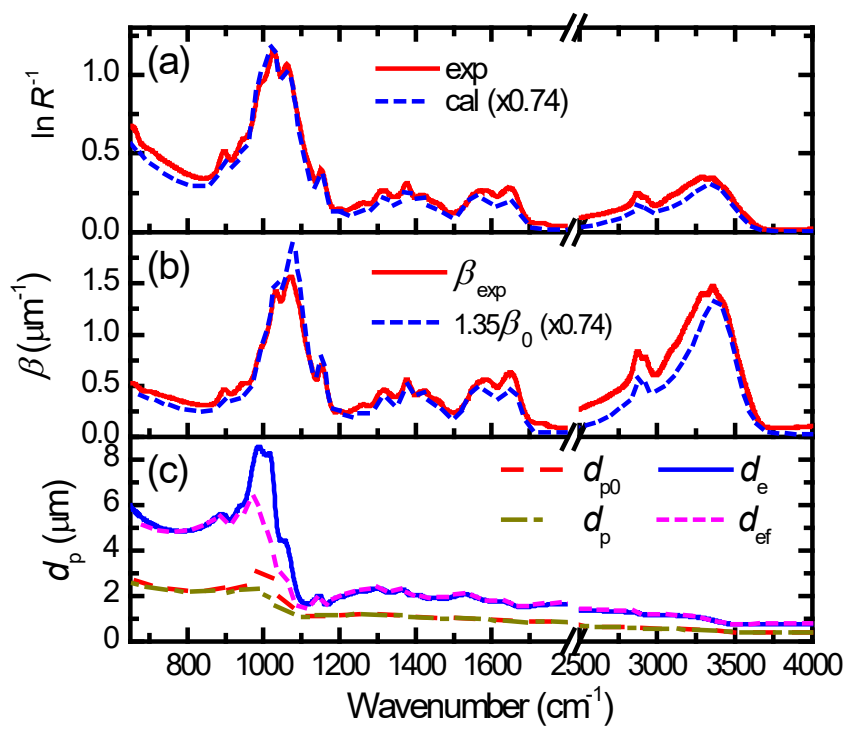

Fig. 2. (a) $\ln R^{-1}$ spectra of experimental $R$ and data calculated with Eq. (5) at $\theta=49^{\circ}$ for a diamond-chitosan interface. The scaling is explained 
in the text. (b) Effective ATR absorption coefficient $\beta \beta_{\exp }=2 \ln R_{\exp }{ }^{-1} / d_{\mathrm{p}}$. (c) Penetration depths and effective thicknesses from Eqs. (1), (4), (8), and the average of Eqs. (2) and (3). For clarity, data in the range 1800-2500 $\mathrm{cm}^{-1}$ are omitted as there are no bands of chitosan in that spectral range.

An example which shows stronger absorption than the two previous examples is ATR measurements on glass. The complex dielectric function of a soda lime glass slide (Corning 2947) was determined by spectroscopic ellipsometry (Fig. S7 in Supplement 1). Figure 3(a) shows the experimental and calculated $\ln R^{-1}$ spectra of the diamond-glass interface in the spectral range of strong absorption in glass 650-1400 $\mathrm{cm}^{-1}$. It can be noticed that there is a very good agreement between the two spectra with scale factor 0.8 accounting for the quality of optical contact. The effective ATR absorption $\beta_{\exp }$ is shown in Fig, 3 (b). It can be noticed that as the strength of absorption increases in the order waterchitosan-glass, the larger the deviation of the line shape of $\ln R^{-1}$ and $\beta$. For the diamond-glass interface the series approximation of Eq. (10) no longer holds due to the strong absorption. However, using a crystal of larger refractive index as Ge $\left(n_{a}=4.0\right)$, the maximum value of $u$ for the Ge-glass interface is about 0.4 . This fact makes it suitable to use the three-term approximation in Eq. (11) with mean values $f=0.2$ and $s=1.5$ (Fig. S8 in Supplement 1) and, in good approximation, $\beta \approx 0.6 \beta$.

As can be seen in Fig. 3(c) the values of $d_{\mathrm{p} 0}$ and $d_{\mathrm{e}}$ diverge and become indetermined in the range $800-1000 \mathrm{~cm}^{-1}$ because $n_{\mathrm{a}} \sin \theta<n$ in Eq. (1). On the other hand, $d_{\mathrm{p}}$ and $d_{\mathrm{ef}}$ show a well-behaved spectral dependence because Eq. (4) ensures a smooth behavior with wavenumber and angle of incidence (Fig. S9 in Supplement 1). At about $870 \mathrm{~cm}^{-1}$ where glass is weakly absorbing, $d_{\mathrm{p}}$ and $d_{\mathrm{ef}}$ reach values of tens of microns.

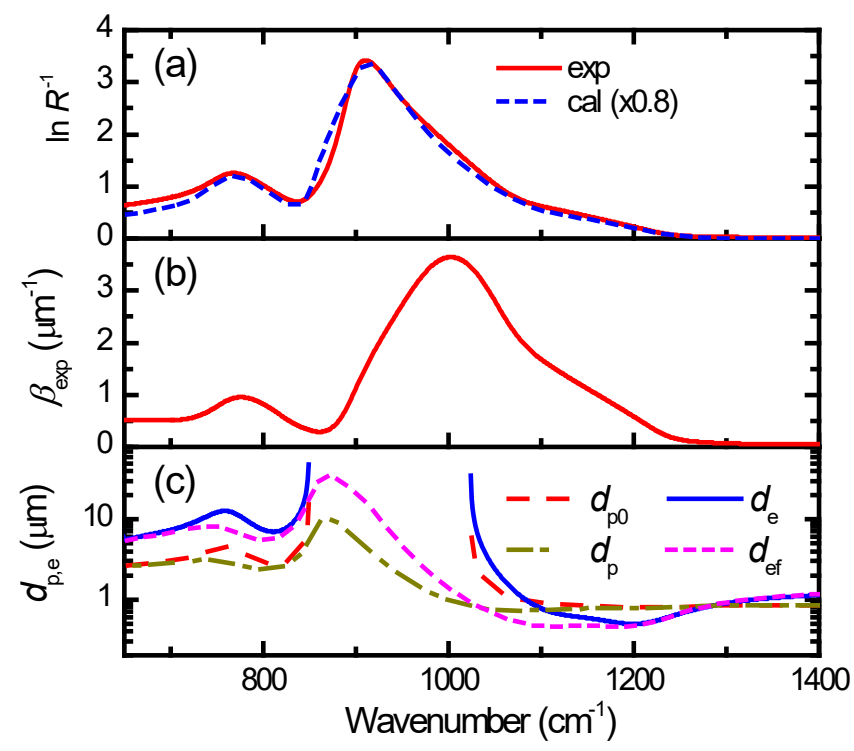

Fig. 3. (a) $\ln R^{-1}$ spectra of experimental $R$ and data calculated with Eq. (5) at $\theta=49^{\circ}$ for a diamond-glass interface in the spectral range of strong absorption of glass. The scaling is explained in the text. (b) Effective ATR absorption coefficient $\beta_{\exp }=2 \ln R \exp ^{-1} / d_{\text {p. }}$. (c) Penetration depths and effective thickness from Eqs. (1), (4), (8), respectively, and average of Eqs. (2) and (3).

In summary, we have developed a general description of ATR spectra and shown that the interpretation for bulk materials might be two-fold. One approach is to use an effective absorption coefficient $\beta$ and the penetration depth $d_{\mathrm{p}}$ for absorbing materials. Another interpretation considers the absorption coefficient and the generalized effective thickness for arbitrary absorption. Experimental data for the interface of diamond with representative materials (water, chitosan, and glass) have been used to demonstrate the reliability of the approach.

\section{Funding sources and acknowledgments.}

Acknowledgments. J. G. Méndez-Lara acknowledges Conacyt the scholarship (\#589937) for doctoral studies. K. Järrendahl acknowledges The Swedish Government Strategic Research Area in Materials Science on Functional Materials at Linköping University (Faculty Grant SFO-Mat-LiU 2009-00971). A. B. Domínguez-Gómez is acknowledged for preparation of the chitosan thick sample.

Disclosures. The authors declare no conflicts of interest.

See Supplement 1 for supporting content.

\section{References}

1. J. Fahrenfort, Spectrochim. Acta 17, 698 (1961).

2. W. N. Hansen, Spectrochim. Acta 21, 815 (1965).

3. N. J. Harrick and F. K. du Pré, Appl. Opt. 5, 1739 (1966).

4. E. Goormaghtigh, V. Raussens, and J.-M. Ruysschaert, Biochim. Biophys. Acta 1422, 105 (1999).

5. M. P. Corujo, M. Sklepari, D. L. Ang, M. Millichip, A. Reason, S. C. Goodchild, P. Wormell, D. P. Amarasinghe, V. Lindo, N. P. Chmel, and A. Rodger, Chirality 30, 957 (2018).

6. V. A. Lorenz-Fonfria, Chem. Rev. 120, 3466 (2020).

7. A. Gaigneaux and E. Goormaghtigh, Analyst 138, 4070 (2013).

8. D. A. Woods and C. D. Bain, Soft Matter 10, 1071 (2014).

9. C. L. Song and S. G. Kazarian, Analyst 144, 2954 (2019).

10. H. Tiernan, B. Byrne, and S. G. Kazarian, Spectrochim. Mol. Biomol. Spectrosc. 241, 118636 (2020).

11. L. A. Averett, P. R. Griffiths, and K. Nishikida, Anal. Chem. 80, 3045 (2008).

12. G. Müller, K. Abraham, and M. Schaldach, Appl. Opt. 20, 1182 (1981).

13. J. E. Bertie and H. H. Eysel, Appl. Spectrosc. 39, 392 (1985).

14. J. B. Huang and M. W. Urban, Appl. Spectrosc. 46, 1666 (1992).

15. M. Boulet-Audet, T. Buffeteau, S. Boudreault, N. Daugey, and M. Pézolet, J. Phys. Chem. B 114, 8255 (2010).

16. J. Aufort, L. Ségalen, C. Gervais, C. Brouder, and E. Balan, Phys. Chem. Minerals 43, 615 (2016).

17. M. Rinaudo, Prog. Polym. Sci. 31, 603 (2006).

18. J. Kumirska, M. Czerwicka, Z. Kaczyński, A. Bychowska, K. Brzozowski, J. Thöming, and P. Stepnowski, Marine Drugs 8, 1567 (2010).

19. J. Brugnerotto, J. Lizardi, F. Goycoolea, W. Argüelles-Monal, J. Desbrières, and M. Rinaudo, Polymer 42, 3569 (2001).

20. G. M. Hale and M. R. Querry, Appl. Opt. 12, 555 (1973).

21. R. A. Mauricio-Sánchez, R. Salazar, J. G. Luna-Bárcenas, and A. Mendoza-Galván, Vib. Spectrosc. 94, 1 (2018).

22. A. B. Domínguez-Gómez, R. A. Mauricio-Sánchez, and A. Mendoza-Galván, Opt. Mater. 84, 564 (2018).

23. S. Ekgasit and A. Padermshoke, Appl. Spectrosc. 55, 1352 (2001). 


\section{References with titles}

1. J. Fahrenfort, "Attenuated total reflection- A new principle for the production of useful infra-red reflection spectra of organic compounds," Spectrochim. Acta 17(7), 698-709 (1961).

2. W. N. Hansen, "Expanded formulas for attenuated total reflection and the derivation of absorption rules for single and multiple ATR spectrometer cells," Spectrochim. Acta, 21(4), 815-833 (1965).

3. N. J. Harrick and F. K. du Pré, "Effective thickness of bulk materials and of thin films for internal reflection spectroscopy," Appl. Opt. 5(11), 17391743 (1966).

4. E. Goormaghtigh, V. Raussens, and J.-M. Ruysschaert, “Attenuated total reflection infrared spectroscopy of proteins and lipids in biological membranes," Biochim. Biophys. Acta 1422(2), 105-185 (1999).

5. M. P. Corujo, M. Sklepari, D. L. Ang, M. Millichip, A. Reason, S. C. Goodchild, P. Wormell, D. P. Amarasinghe, V. Lindo, N. P. Chmel, and A. Rodger, "Infrared absorbance spectroscopy of aqueous proteins: Comparison of transmission and ATR data collection and analysis for secondary structure fitting," Chirality 30(8), 957-965 (2018).

6. V. A. Lorenz-Fonfria, "Infrared difference spectroscopy of proteins: From bands to bonds," Chem. Rev. 120(7), 3466-3576 (2020).

7. A. Gaigneaux and E. Goormaghtigh, "A new dimension for cell identification by FTIR spectroscopy: depth profiling in attenuated total reflection," Analyst 138, 4070-4075 (2013).

8. D. A. Woods and C. D. Bain, "Total internal reflection spectroscopy for studying soft matter," Soft Matter 10(8), 1071-1096 (2014).

9. C. L. Song and S. G. Kazarian "Three-dimensional depth profiling of prostate tissue by micro ATR-FTIR spectroscopic imaging with variable angles of incidence," Analyst, 144(9), 2954-2964 (2019).

10. H. Tiernan, B. Byrne, and S. G. Kazarian, "ATR-FTIR spectroscopy and spectroscopic imaging for the analysis of biopharmaceuticals," Spectrochim. Acta Mol. Biomol. Spectrosc. 241, 118636 (2020).

11. L. A. Averett, P. R. Griffiths, and K. Nishikida, "Effective path length in attenuated total reflection spectroscopy," Anal. Chem. 80, 3045-3049 (2008).

12. G. Müller, K. Abraham, and M. Schaldach, "Quantitative ATR spectroscopy: some basic considerations," Appl. Opt. 20(7), 1182-1190 (1981).

13. J. E. Bertie and H. H. Eysel, "Infrared intensities of liquids I: Determination of infrared optical and dielectric constants by FT-IR using the CIRCLE ATR cell," Appl. Spectrosc. 39(3), 392-401 (1985).

14. J. B. Huang and M. W. Urban, "Evaluation and analysis of attenuated total reflectance FT-IR spectra using Kramers-Kronig transforms," Appl. Spectrosc. 46(11), 1666-1672 (1992).

15. M. Boulet-Audet, T. Buffeteau, S. Boudreault, N. Daugey, and M. Pézolet, "Quantitative determination of band distortions in diamond attenuated total reflectance infrared spectra," J. Phys. Chem. B 114(24), 8255-8261 (2010).

16. J. Aufort, L. Ségalen, C. Gervais, C. Brouder, and E. Balan, "Modeling the attenuated total reflectance infrared (ATR-FTIR) spectrum of apatite," Phys. Chem. Minerals 43, 615-626 (2016).

17. M. Rinaudo, "Chitin and chitosan: Properties and applications," Prog. Polym. Sci. 31(7), 603-632 (2006).

18. J. Kumirska, M. Czerwicka, Z. Kaczyński, A. Bychowska, K. Brzozowski, J. Thöming, and P. Stepnowski, "Application of spectroscopic methods for structural analysis of chitin and chitosan," Marine Drugs, 8(5), 15671636 (2010).

19. J. Brugnerotto, J. Lizardi, F. Goycoolea, W. Argüelles-Monal, J. Desbrières, and M. Rinaudo, "An infrared investigation in relation with chitin and chitosan," Polymer 42(8), 3569-3580 (2001).

20. G. M. Hale and M. R. Querry, "Optical Constants of Water in the 200nm to 200- $\mu$ m, " Appl. Opt. 12(3), 555-563 (1973).

21. R. A. Mauricio-Sánchez, R. Salazar, J. G. Luna-Bárcenas, and A. Mendoza-Galván, "FTIR spectroscopy studies on the spontaneous neutralization of chitosan acetate films by moisture conditioning," Vib. Spectrosc. 94, 1-6 (2018).

22. A. B. Domínguez-Gómez, R. A. Mauricio-Sánchez, and A. MendozaGalván, "Extinction coefficient of free-standing chitosan films determined from partially coherent transmittance spectra," Opt. Mater. 84, 564-571 (2018).

23. S. Ekgasit and A. Padermshoke, "Optical contact in ATR/FT-IR spectroscopy," Appl. Spectrosc. 55(10), 1352-1359 (2001). 